\title{
INFOSHARE - An Information Sharing Tool for Public Health during the 2009 Presidential Inauguration and $\mathrm{H} 1 \mathrm{~N} 1$ Outbreak
}

\author{
Wayne Loschen ${ }^{1}$, Richard Seagraves ${ }^{1}$, Rekha Holtry ${ }^{1}$, Lang Hung ${ }^{1}$, Joesph Lombardo ${ }^{1}$, \\ Sheri Lewis ${ }^{1}$ \\ ${ }^{1}$ Johns Hopkins University, Applied Physics Laboratory
}

\begin{abstract}
The 2009 Presidential Inauguration and H1N1 outbreak called for real-time electronic information-sharing and surveillance across multiple jurisdictions to better understand the health of migrating populations. The InfoShare web application proved to be an efficient tool for users to share disease surveillance information. During both high profile events, public health users shared information within a secure access-controlled website across regions in the U.S. and among agencies. Due to its flexible design, InfoShare was quickly modified from its 2009 Inauguration interface to an interface that supports H1N1 surveillance. Through discussions and post-use surveys, a majority of InfoShare users revealed that the tool had provided a valuable and needed function. InfoShare allowed individual jurisdictions to receive timely and useful information, which, when merged with neighboring jurisdictions, significantly enhanced situational awareness for better decision-making and improved public health outcomes.
\end{abstract}

\section{Background}

Public health has conducted enhanced disease surveillance activities during high profile events for many years. The utilization of emergency operations centers, aid stations, and increased staffing for political events or large gatherings permits public health officials to keep an up-todate picture of the community's health during these high profile events; this enhanced level of knowledge is also known as situational awareness. A real-time electronic disease surveillance system may provide utility in monitoring the health of a population during mass gatherings or special events. In special cases, the event will affect multiple jurisdictions [1]. Information or data sharing among jurisdictions provides an understanding of the health of the migrating populations. Large-scale public events with attendees from multiple regions, such as the Super Bowl and the Olympics, are examples of events can that can benefit from cross-jurisdictional electronic surveillance to assess the health risks for the gathered and returning populations [2]. This article describes two recent multi-regional events that used information-sharing technology to enhance the disease surveillance capability: The 2009 Presidential Inauguration and the 2009 H1N1 influenza pandemic. 
The 2009 U.S. Presidential Inauguration attracted an estimated 1.8 million people to the National Capital Region (NCR) of Maryland, Virginia, and the District of Columbia [3]. This mass gathering event called for ongoing public health awareness across the regions where Inaugural activities occurred. Similarly, the H1N1 outbreak in spring 2009 resulted in increased influenzalike infection activity within the U.S. and throughout the world [4], leading the World Health Organization (WHO) to declare a pandemic of widespread human infection [5]. As a result, public health departments across the U.S. continually monitored the health of their communities. These two events brought to light the need for real-time electronic information-sharing and surveillance across multiple jurisdictions.

\section{Methods}

\section{Sharing Information v. Sharing Data}

Based on the types of public health disease information that is used for community disease surveillance, patient health-indicator data can be categorized in two fundamental ways: data and information. Raw line-listings, such as hospital emergency department chief complaint records, are considered "data" because they are original facts. Data in original form, after removal of duplicate records or erroneous entries, or when aggregated by particular attributes can be associated with individual patients with minimal or no effort. On the other hand, derived outputs such as univariate and multivariate statistical analysis outputs from aberration-detection systems and epidemiological interpretations are considered "information." Here, the output is not listed at an individual level and the information is no longer traceable to patient records. Because "data" are gathered at the patient-level, depending on the amount of information required on each patient, they can be potentially difficult to attain and share due to regional/institutional policies and laws that protect individual privacy [6]. However, "information" refers to summaries, opinions, or conclusions about data, and therefore may be less challenging to attain and more suited for sharing across jurisdictions and agencies to enhance situational awareness.

Sharing information instead of data provides several advantages. First, sharing information essentially sharing statistical outputs or opinions about situations - is less restrictive and requires simpler agreements. Second, when considering disease surveillance that spans across jurisdictions, interpretations of public health data anomalies by local users are more likely to provide better-informed explanation of findings because of detailed knowledge of the data and baseline disease patterns in the community they serve. If local users provide short interpretations of disease surveillance findings, users in other jurisdictions can quickly review events and focus on relevant material without having to duplicate efforts. Moreover, sharing information can provide an increased sense of collaboration and trust among communities. As informationsharing among users across jurisdictions becomes commonplace, beneficial partnerships can be formed that promote exchange of new ideas, successful protocols and practices, diseasemonitoring techniques, and tools related to disease surveillance.

On the other hand, there are some disadvantages to sharing information. Sharing information instead of data results in loss of specificity. Specificity of the data inputted into anomalydetection algorithms determines their validity. Because of the non-specific and processed nature of "information," statistical algorithms have reduced capability for anomaly detection. It would be possible, however, to use shared information to fine-tune alerting algorithms. For example, if information is shared about a possible respiratory disease outbreak in a particular jurisdiction, the 
adjacent jurisdictions may be able to increase sensitivity in local respiratory disease detectors to capture similar cases. The heightened awareness would be useful in prompting public health officials in neighboring or epidemiologically-linked regions to investigate a local event more closely; however, they will be unable to distinguish records that led to the alert beyond the summaries or interpretations provided in the shared information.

\section{InfoShare}

InfoShare is a tool developed by The Johns Hopkins University Applied Physics Laboratory (JHU/APL) that allows public health officials to share concerns and preliminary investigation results among multiple jurisdictions and agencies. The tool is designed such that messages/information being shared adequately relay the situation without requiring aggregated or identifiable data to be exposed, yet allowing the user to enter more detailed information if needed to further describe the event. The model promotes the entry of information into structured fields to enable computer readability. This allows the information to be both viewed visually and used systematically by algorithms, decision support, and messaging tools. Moreover, the computer readable fields can be automatically populated by other systems to help users generate messages easily and maintain control on the level of information shared.

\section{InfoShare Architecture}

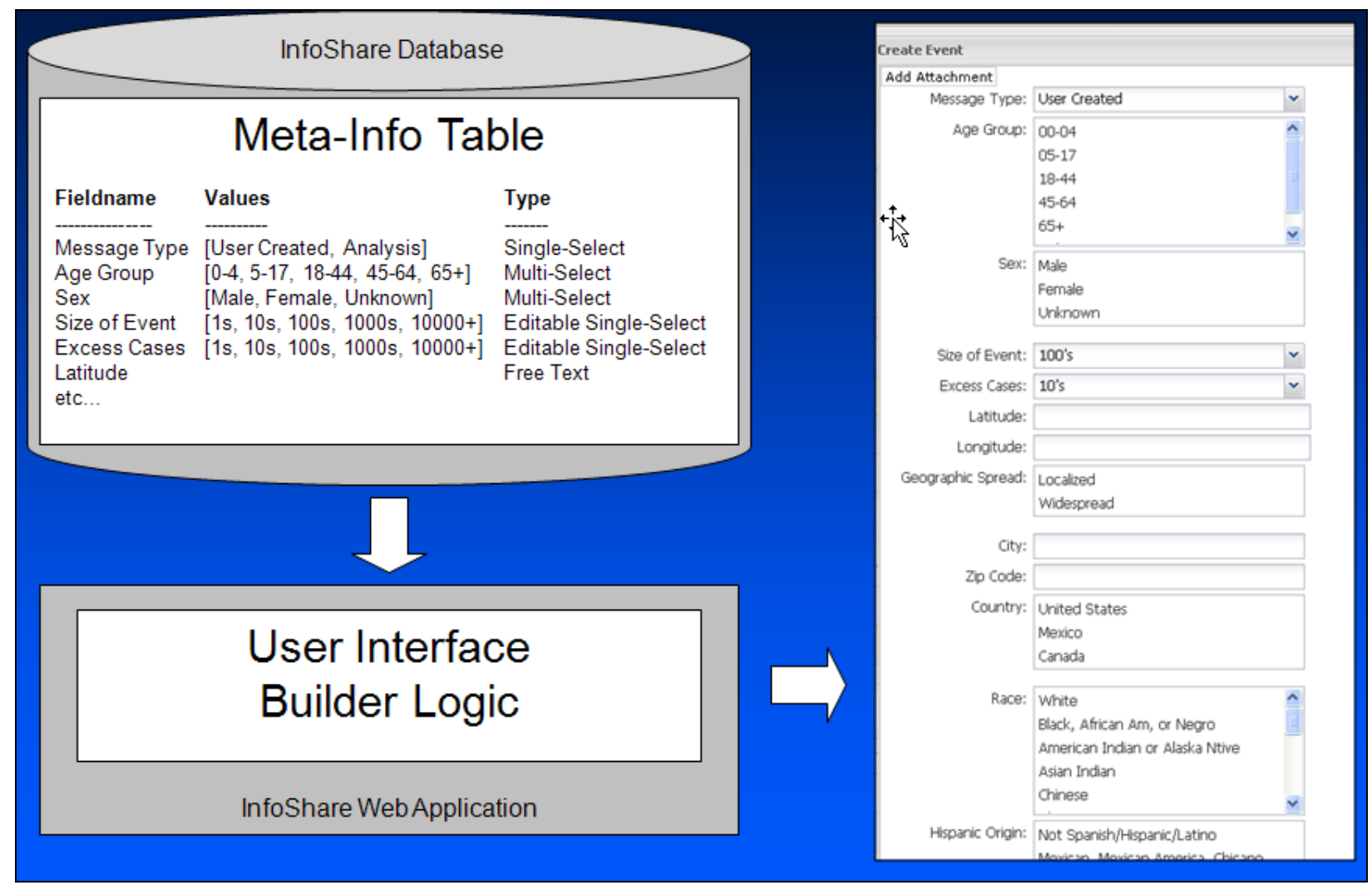

Figure 1. The InfoShare architecture consists of two main components: the database and web application. The InfoShare database holds the meta-information table, which defines fields 
included in the current web application. The inaugural instance included fields of message type, age group, sex, size of event, excess cases, latitude, etc.

The InfoShare web application enables posting of structured fields by allowing users to select the appropriate single or multiple values from drop-down or list boxes. Unstructured fields also enable users to enter free text to fully describe the event and to facilitate discussion on the events. Users can also attach files (e.g., images, documents, etc.) to the posted event. All information on this web application is communicated securely over Secure Socket Layer (SSL).

\section{InfoShare Web Interface Create Event Form}

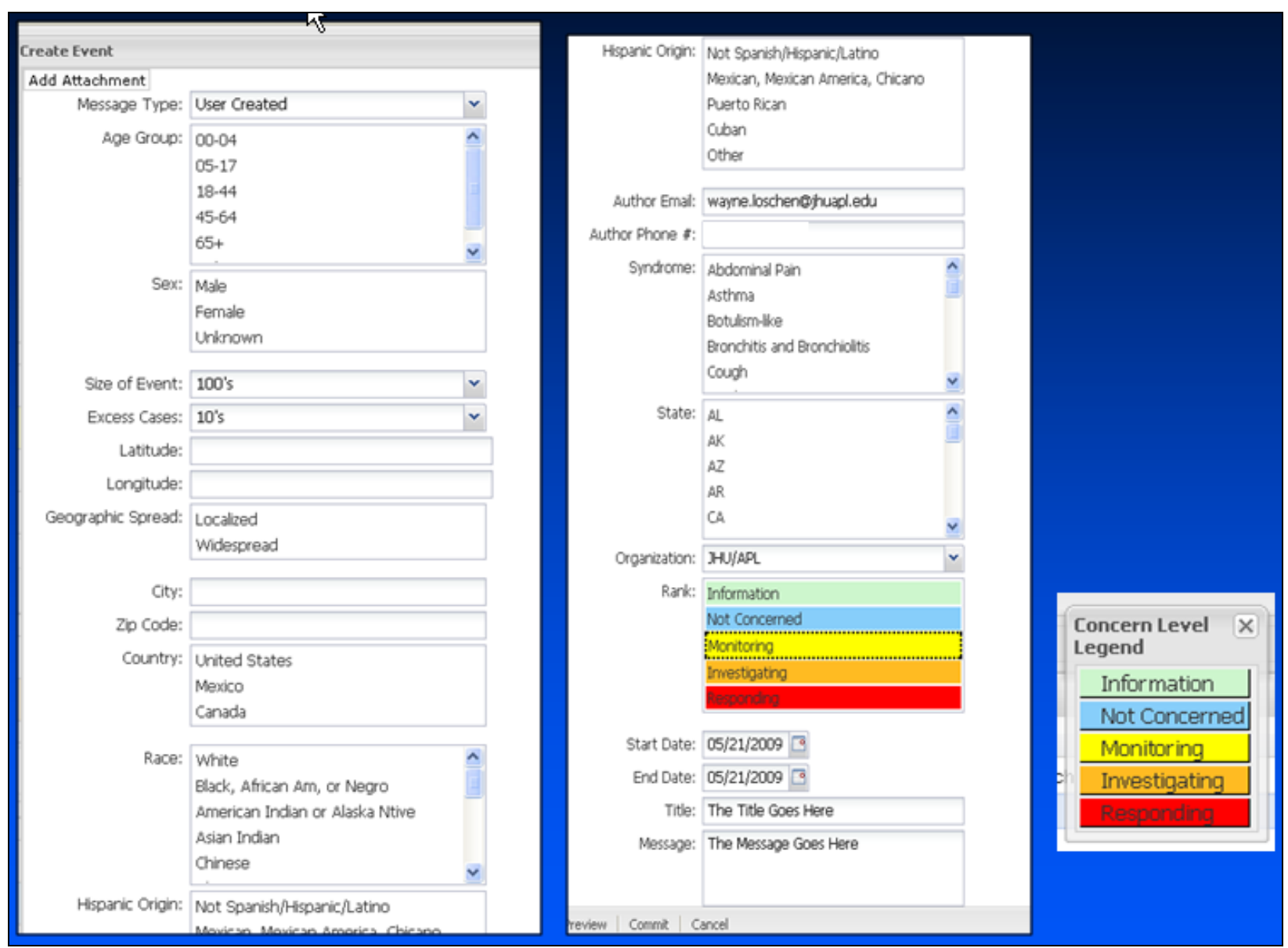

Figure 2. The InfoShare web interface allows end users to enter data related to the event using structured and unstructured fields.

It is important to note that the InfoShare web interface allows end users to rank the concern level of the event. There are currently five ranks for each event (Figure 3 ). 


\section{Responding}

identified or is highly likely

Investigating - Investigating potential outbreak/new disease-clusters

Monitoring - Closely monitoring some unusual patterns noted in data

Not Concerned - No unusual patterns noted in data; No action suggested

Info - For information purposes only

Figure 3. The InfoShare web interface allows users to rank the level of concern for the event. There are five rankings, ranging from high magnitude outbreaks to informational purposes only.

\section{Linkage within ESSENCE}

In recent years, it has become increasingly common for public health entities to use electronic disease surveillance systems that employ health-indicator data for timely recognition of events of public health significance and for tracking disease trends across time. The civilian Electronic Surveillance System for the Early Notification of Community-based Epidemics (ESSENCE) is one such system used by some U.S. public health departments [7]. To assist with the disease surveillance and outbreak investigations, these systems contain multiple statistical alerting and data mining capabilities. In addition to automated alerting, ESSENCE users in particular have the ability to write custom queries of varying complexity into databases for dynamic processing. If a statistical alert is noted, ESSENCE users have the ability to view data at a detailed level to characterize the alert more thoroughly and to interpret findings in its proper context. The end product of conducting electronic disease surveillance is a wealth of timely public health information. Because community disease patterns and outbreaks are not confined by geographic boundaries, this derived information can be useful to jurisdictions with primary responsibility, and sometimes to adjacent regions and larger jurisdictions as well. With this mindset, InfoShare was tested among ESSENCE users in spring of 2009 to facilitate simplified information-sharing across public health agencies. To accomplish this, the InfoShare website was made accessible via two methods. Within ESSENCE, "share" buttons were added on specific alerts that enabled users to easily share information about potential health risks (See Figure 4). This allowed users to create InfoShare messages directly from ESSENCE with most of the messages pre-filled with information. Alternatively, authorized users also had the option of accessing the InfoShare website directly to post and read information.

In addition to the website modifications, users in the NCR developed a set of specific queries that they agreed to monitor during the heightened surveillance period. These shared queries utilized the "Advanced Query Tool" feature of ESSENCE and utilized free-text chief complaint searches to look for specific diseases such as meningitis, hypothermia, dehydration, and others. 


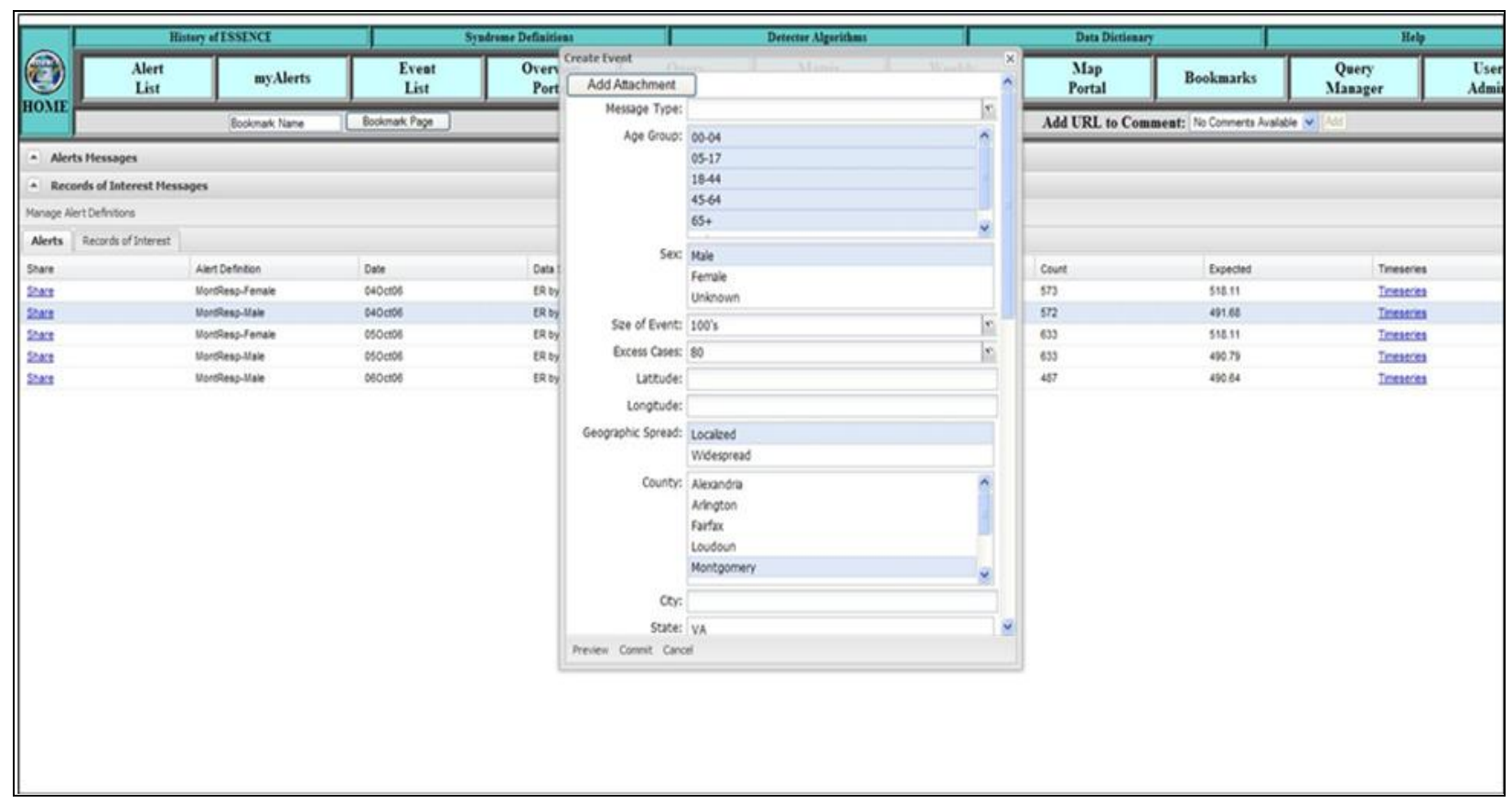

Figure 4. InfoShare was linked to ESSENCE with the addition of a new "Share" feature.

\section{InfoShare Use During the 2009 Inauguration}

Ten days before and after the 2009 Presidential Inauguration, public health officials in Maryland, Virginia, the District of Columbia, and the Centers for Disease Control and Prevention (CDC) participated in information-sharing using InfoShare. NCR users monitored their respective populations using emergency room chief complaints, over-the-counter drug sales, aid station reports, poison control reports, and school absenteeism in ESSENCE [8]. CDC users monitored the NCR by reviewing military office visits, Veteran's Affairs office visits, emergency room chief complaints, and laboratory data via the national BioSense surveillance system. CDC users had the ability to securely log into the Information Sharing website to post messages regarding potential health risks they discovered. Users from both systems were able to view and share additional findings using this model for information sharing in a prototype message exchange system (See Figure 3) [9].

The web interface supported secure communications over SSL and provided users with the ability to: 1) create events and comments using structured and unstructured fields; and 2) attach additional files (e.g., images and documents). The style of the interface mimicked Microsoft Outlook and allowed users to view existing events in the upper-right corner, filter those events using the left-side, and view the contents of the selected event in the bottom-right corner (See Figure 5). Users had the ability to provide comments to listed events and include their own concern level to an event. Users could filter the events by any parameter and view a description of the filtered parameters. These quick filters were included on the left-side bar by syndrome categories: Botulism-Like, Fever, Gastrointestinal (GI), Hemorrhagic-Illness, Localized Lesions, Lymphadenitis, Neurological, Other, Rash, Respiratory, and Sudden Illness/Death. The 
aforementioned syndromes were predetermined by expert consensus and may be influenced by several factors such as data source characteristics, surveillance focus area, public health practitioner/agency priorities, etc [10].

\section{Inaugural Web Interface}

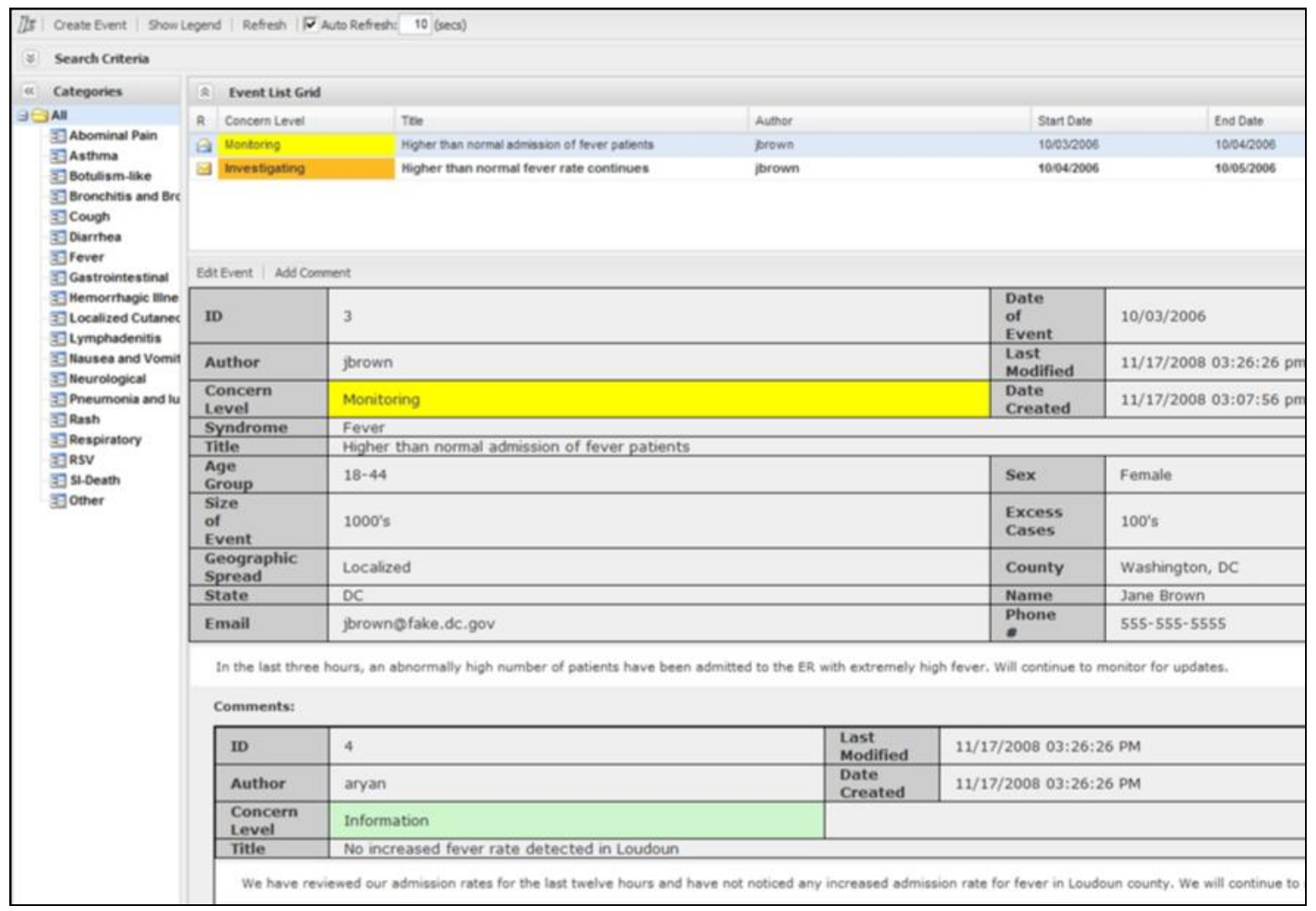

Figure 5. The InfoShare Inaugural web interface allows users to view events posted by other public health officials.

\section{InfoShare for H1N1 surveillance}

When the H1N1 outbreaks occurred in spring of 2009, the InfoShare system used for the Presidential Inauguration was easily transitioned for use in H1N1 surveillance. Due to the flexible design of InfoShare, in less than an hour the application was adjusted to support H1N1 surveillance. Along with other changes in the original data fields, the web interface was modified to provide quick filters by organizations instead of diseases. In addition, a user registration capability was enabled to help handle the influx of new users (See Figure 6). 
InfoShare for H1N1 Web Interface

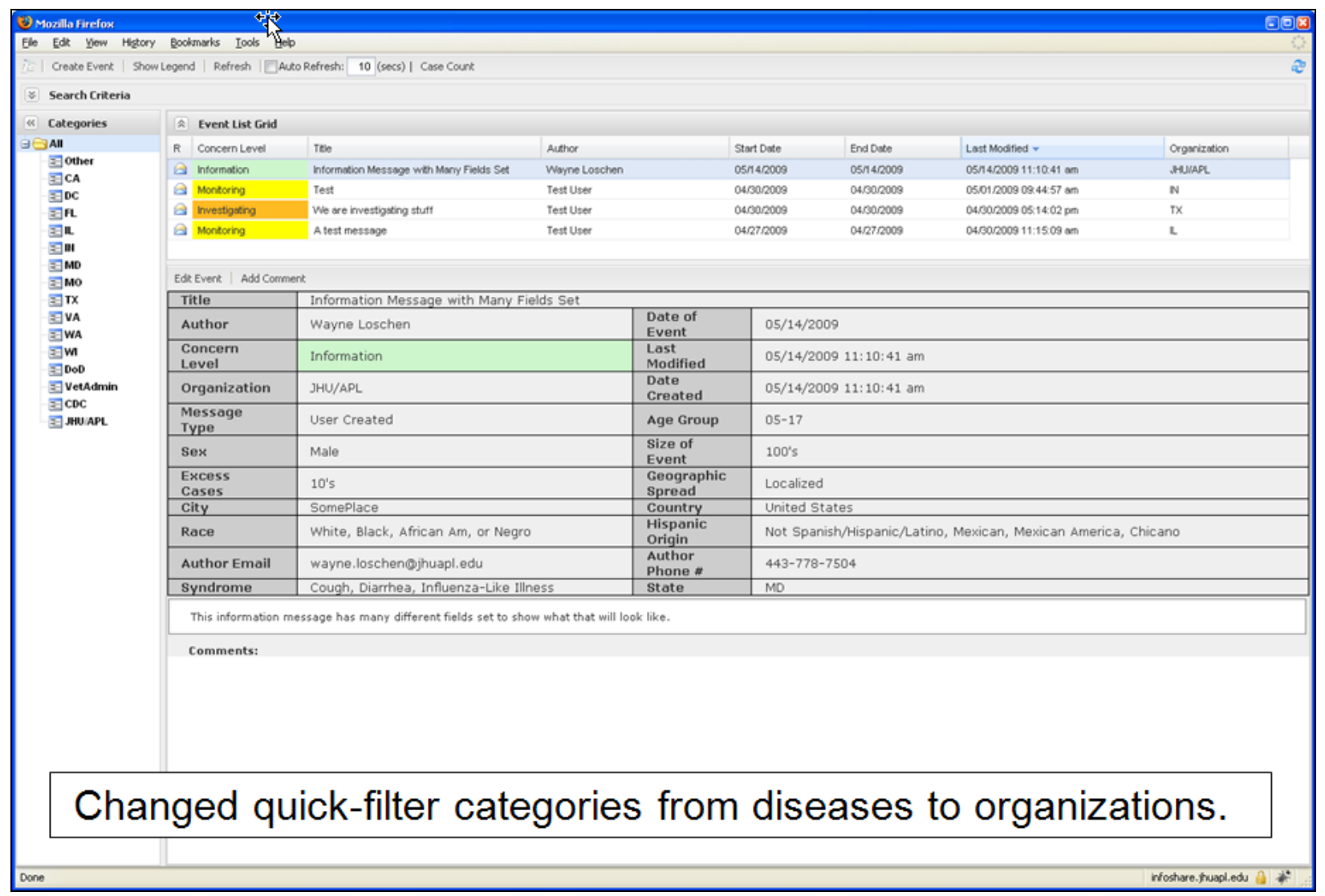

Figure 6. The web interface continued to support the previous information exchange functions with the Quick-filter categories being changed from diseases to organizations, modification of some collected data fields, and the addition of the user registration capability.

An email was drafted and sent to many ESSENCE installations, including: Local Health Departments, State Health Departments, Department of Defense (DoD), Department of Veterans Affairs (VA), and to the CDC. The email described previous InfoShare accomplishments during the Presidential Inauguration, along with the tool's capabilities and protocols for use of the site. Accounts were created for ten states (Wisconsin, Washington, California, Illinois, Indiana, Missouri, Maryland, Virginia, Florida, and Texas), District of Columbia, DoD, VA, and CDC users. 


\section{InfoShare Participants during the 2009 Inauguration}

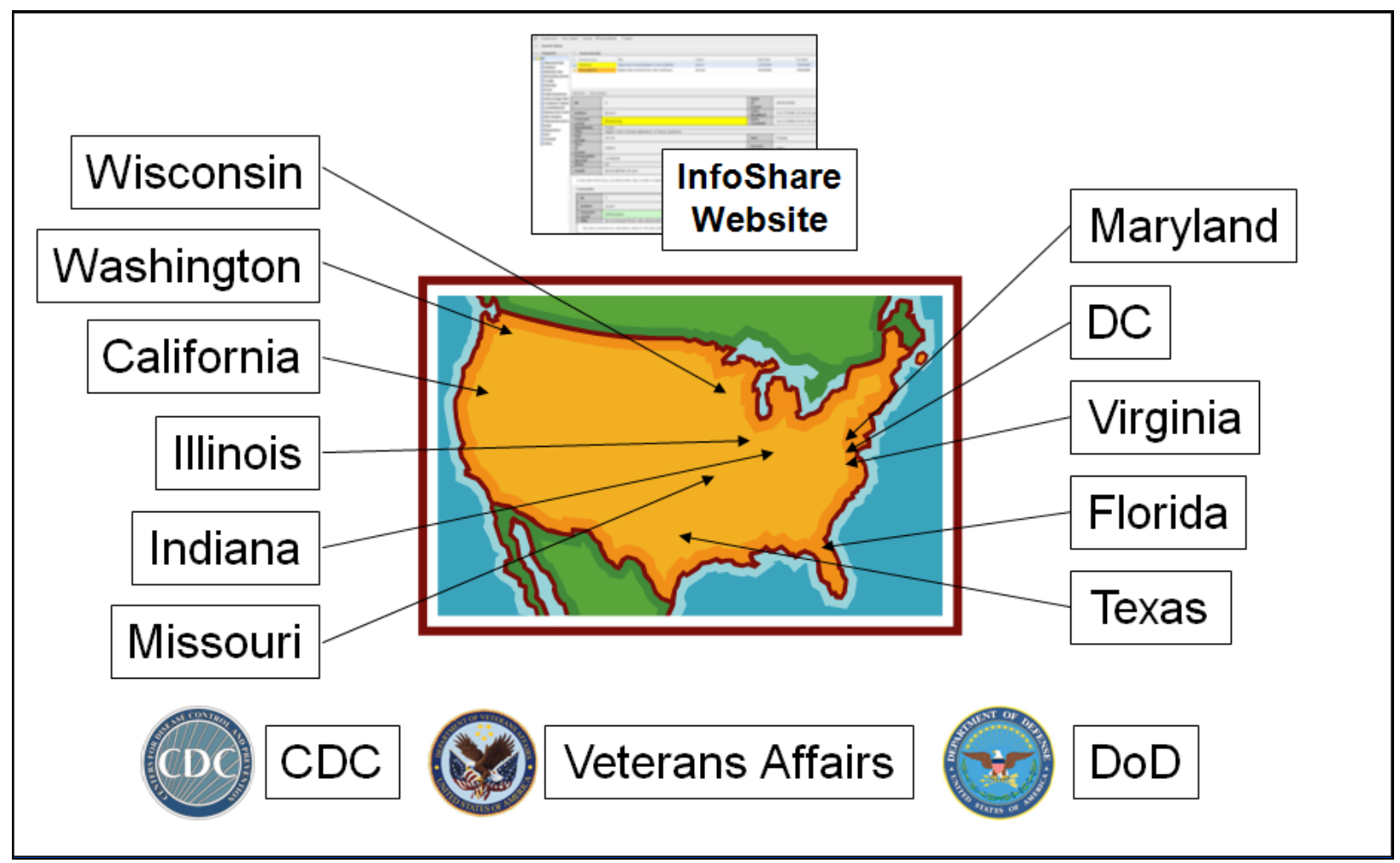

Figure 7. This figure depicts participants nationwide who used InfoShare during the 2009 H1N1 surveillance event.

During both events in which InfoShare was used, a set of collectively accepted protocols were observed by participants:

- Accessing the InfoShare site requires a login and password.

- Information posted to the InfoShare site was only viewable by collaborating partners.

- The information contained in a posting was confidential, to be used only by collaborating partners to perform authorized public health functions.

- Prior to sharing any information with a public health or other entity outside the collaborative group, the posting was first to be verified or clarified by contacting the author and authorized by the public health agency.

- Information posted on the website was provided for informational purposes only; users were required to recognize that professional interpretations could vary.

\section{Results}

With use of InfoShare during the 2009 Presidential Inauguration period, 33 events and 12 additional comments were created. Of these events, 30 had a concern level of "Not Concerned" or "Information," the remaining 3 events were "Monitoring." During this 21 day period, the local ESSENCE system was monitoring the same 10 geographic regions, 10 syndromes, and 7 age groups of interest, amounting to 14,700 possible statistical alerts. During this time, 
ESSENCE generated 886 statistical alerts (6\% alert rate); 314 were red alerts (representing the highest concern level of an automated detector) and the remaining 572 were yellow warnings (representing a lower concern level). This means that comments were generated on InfoShare for $0.3 \%$ of the alerts generated by ESSENCE.

Completion of a user's survey determined that about $63 \%(10 / 16)$ of users utilized the shared queries at least once a day. About 94\% (15/16) of InfoShare users felt the system had value. Approximately $70 \%$ (7/10) felt a need for formal inter-agency policies and $75 \%(12 / 16)$ were able to access useful information.

The H1N1 InfoShare site had 14 events created between 4/30/2009 to 6/25/2009. Ten of these events were considered "Information," two were "Monitoring," and two were "Investigating." In addition to the events, there were five comments associated with those events. No formal survey was conducted during or after the H1N1 event.

\section{Discussion}

There were many lessons learned from using the InfoShare system during the Inauguration and H1N1 emergence of 2009. First, it became evident that posting events must be an easy process for public health practitioners. It was important for one to be able to disperse information easily in a time of urgent need. Many users were interested in the speedy posting process of the "share" feature because information was pre-populated automatically. Second, a protocol document - serving as a reference for users describing the methods for reviewing, posting, and sharing information on the site - was critical. This became the most important factor when the InfoShare site was moved from the initial trial during the Inauguration to the secondary use of the H1N1 outbreak. Having a set of formal rules of use gave many users a higher comfort level with posting initial impressions to users outside their jurisdictions. Third, sharing query definitions were found to be valuable during events. In addition, many users found the added communication with regard to outbreaks, whether it be current or potential, to be extremely valuable. This enabled InfoShare users to work efficiently, without having to duplicate efforts. Another finding was that the InfoShare system needed to accommodate non-specific and specific information fields. This capability was required within the "Size of Event" and "Excess Cases" fields, which allowed the user to input the magnitude of the event in question. Some users wanted to report specific numbers in these fields, while others preferred non-specific reporting. To accommodate both preferences, the user interface provided pull down choices such as 1 's, 10 's, 100's, 1,000's, 10,000+, and also allowed the user to type in their own value.

During the H1N1 activity period, many users of the H1N1 InfoShare application were also involved with the DiSTRIBuTE project from the International Society of Disease Surveillance [11]. The DiSTRIBuTE project used an email-based listserv for communication. During this period of time, DiSTRIBuTE listserv usage increased and a large amount of information was shared between everyone on the listserv. When the issue of using non-secure email to share public health data was raised, some users acknowledged this limitation and admitted that a tool such as InfoShare would have been a safer choice. Regardless, the popularity gained by DiSTRIBuTE during H1N1 and the extent to which it was used highlights the following: ease-ofuse of the information-sharing tool - coupled with its incorporation into day-to-day work practices - is paramount to successful implementation. When information sharing across 
jurisdictions is purely voluntary, it is difficult for a system such as InfoShare to compete with the simplicity of email-based systems, despite known dangers from information loss and theft inherent to such communications.

\section{Conclusion}

Mass gathering and large event health surveillance are multi-jurisdictional challenges. Individual jurisdictions are receiving timely and valuable information using real-time electronic disease surveillance systems, that when merged with neighboring jurisdictions, can significantly enhance situational awareness. This heightened awareness can lead to better decision-making and improved public health outcomes.

The InfoShare web application was developed by JHU/APL and proved to be an efficient tool for users to share disease surveillance information. Public health users shared information within a secure access-controlled website across regions in the U.S. and among agencies. Several features were in place that simplified the creation of structured and unstructured entities. By grouping and displaying postings, thereby making them simple to access and easy to process, the user interfaces were particularly user-friendly. The frequency of InfoShare use during the events discussed was fair. In addition, post-use surveys revealed that the tool provided a valuable and needed function for a majority of the users.

However, reviewing the use of a separate email-based data-sharing tool deployed during the H1N1 outbreaks revealed that InfoShare cannot compete with standard email for familiaritybased ease-of-use and simplicity. It was evident also that because of its public health eventfocused design to collect, organize, and display postings, as well as its capability for capturing information in structured and unstructured form, fitting metrics must be used to gauge InfoShare's value for disease surveillance.

More importantly, the InfoShare application provided a safe forum for sharing disease surveillance information, which if stolen or misused from an insecure environment could have serious consequences. Finally, use of InfoShare during these public health events brought to light the need for statistical alerting capability of shared postings. Hence, future enhancements should include automated analysis of the information posted from standardized and structured fields.

\section{Acknowledgements}

This work was made possible through grant number 1U38HK000062-01 from the US Department of Health and Human Services (HHS) Centers for Disease Control (CDC). Its contents are solely the responsibility of the authors and do not necessarily represent the official views of the CDC. The authors would like to acknowledge the exceptional cooperation of the Maryland Department of Health and Mental Hygiene (DHMH), the Virginia Department of Health, Division of Surveillance and Investigation (VDH/DSI), District of Columbia Department of Health, Bureau of Epidemiology and Health Risk Assessment (DCDOH), the eight local health departments represented in the NCR Enhanced Surveillance Operating Group (ESOG), and the BioIntelligence Center (CDC). 
INFOSHARE - An Information Sharing Tool for Public Health during the 2009 Presidential Inauguration and H1N1 Outbreak

\section{References}

[1] CIFOR. Council to Improve Foodborne Outbreak Response. Special Considerations for Multijurisdictional Outbreaks. www.cste.org/dnn/Portals/0/CIFOR\%20Guidelines\%20Chapter\%207.pdf (last visited May 17, 2010).

[2] Lombardo JS, Sniegoski CA, Loschen WA, Westercamp M, Wade M, Dearth S, Zhang, G. Public health surveillance for mass gatherings. Johns Hopkins APL Technical Digest 2008; 27(4): 347-355.

[3] Published by The Washington Post and available at http://www.washingtonpost.com/wpdyn/content/article/2009/01/21/AR2009012103884.html. (last visited Mar. 10, 2010).

[4] Centers for Disease Control and Prevention (CDC). Update: Influenza Activity-United States, August 30, 2009-January 9, 2010. MMWR. 59(02):38-43.

http://www.cdc.gov/mmwr/preview/mmwrhtml/mm5902a3.htm. (last visited Mar. 19, 2010).

[5] World Health Organization (WHO). World Now at the Start of 2009 Influenza Pandemic. http://www.who.int/mediacentre/news/statements/2009/h1n1_pandemic phase6_20090611/en/in dex.html. (last visited Mar. 19, 2010).

[6] The Health Insurance Portability and Accountability Act of 1996 (HIPAA). http://www.hipaa.org. (last visited June 25, 2010).

[7] Lombardo JS, Buckeridge DL, editors. Disease Surveillance: A Public Health Informatics Approach. Hoboken (NJ): John Wiley \& Sons, Inc.; 2007.

[8] Lombardo JS. The ESSENCE II Disease Surveillance Test Bed for the National Capital Area. Johns Hopkins APL Tech Dig. 2003;24(4):327-334.

[9] Loschen WA. Methods for information sharing to support health monitoring. Johns Hopkins APL Technical Digest 2008; 27(4): 340-346.

[10] Lombardo J, Burkom H, Elbert E, Magruder S, Lewis SH, Loschen W, et al. A Systems Overview of the Electronic Surveillance System for the Early Notification of Community-Based Epidemics (ESSENCE II). J Urban Health. 2003;80(2):i32-i42.

[11] Distribute Project - Influenza Surveillance System. http://isdsdistribute.org/ (last visited June 16, 2010).

\section{Correspondence:}

Wayne, Loschen, MS

wayne.loschen@jhuapl.edu 